Originalveröffentlichung in: Boer, Pim den u.a. (Hrsg.): Europäische Erinnerungsorte, Bd. 2, Das Haus Europa,

München 2012, S. 137-150

\title{
Hubertus Günther Die Gotik als der europäische Baustil
}

„Sie alle kennen die gotische Kathedrale“. Mit dieser Feststellung leitete Volkwin Marg am 21. April 2010 seinen Vortrag zur Eröffnung der Ausstellung „Von Kapstadt bis Brasília“ im Münchner Architekturmuseum ein. Aus Anlass der Fußballweltmeisterschaft in Südafrika wurden die spektakulären Sportstadien der Architekten von Gerkan Marg und Partner präsentiert, einem der größten Planungsbüros der Welt, zu dessen Gründern Marg gehört. Marg bezog sich auf die Gotik, um im Vergleich mit ihr zu erklären, nach welchen Richtlinien die Arenen konzipiert wurden: Wie bei der gotischen Kathedrale sollte die Konstruktion frei von modischer Effekthascherei ganz auf die Funktion ausgerichtet sein, auf praktische Bedürfnisse und technische Bedingungen; das höchste Ziel sei, zeitgemäße Symbolik zum Ausdruck zu bringen. Im weiteren Sinn, ergänzte Marg im persönlichen Gespräch, habe die gotische Kathedrale das gleiche wie die Sportstadien vor Augen geführt: allgemeine „Bereitschaft zur Begeisterung".

Vergleiche, wie sie in dem Vortrag gezogen wurden, sind weit verbreitet. Zur gleichen Zeit las man in einem "Zugbegleiter" der Deutschen Bahn über eines der Fußballstadien für die Weltmeisterschaft in Südafrika: „Was im Mittelalter, was in der Gotik die steinerne Konstruktion der Kathedralen war, stellen heute die stählernen Konstruktionen der weitspannenden Dächer dar“. Auch die Lichtführung in der neuen Lagerhalle des Vitra-Museums in Weil am Rhein (Architekturbüro Sanaa, Tokio), die „fraktale Geometrie“ der Halle des Flughafens Stuttgart (Ing.Büro Frei-Otto, Stuttgart), die Industriebauten des Ruhrgebiets haben jüngst Assoziationen an die Gotik geweckt. Die „Süddeutsche Zeitung“ apostrophierte die 2003-05 von Herzog/de Meuron gebaute Allianz Arena einmal enthusiastisch als „Fußballkathedrale“.

Seitdem „Funktionalismus“ als Leitlinie gilt, seit Peter Behrens, dem Bauhaus und Le Corbusier, werden immer wieder Parallelen zwischen moderner Architektur und der gotischen Kathedrale gezogen. Sie setzen bei den neuen Bautechniken an mit ihrer Konzentration von Lasten oder Zugkräften auf kleine Elemente und reichen bis hin zu den gesellschaftlichen Verhältnissen, die in den Bauten zum Ausdruck kommen. Oswald Spengler schrieb in seinem einst populären Buch vom „Untergang des Abendlandes“ (1923) sogar, die Seele des Abendlandes habe ihr Weltgefühl durch die „Räume gotischer Dome“ ebenso wie durch die „Formeln der Funktionstheorie“ ausgedrückt.

Was die gotische Kathedrale auszeichnet, ist oft im Vergleich mit dem klassischen griechischen Tempel beschrieben worden. Beide wirken wie Solitäre, sie scheinen fast aus der historischen Entwicklung herauszufallen. Vor dem griechischen Tempel sind Steinbauten, die von Säulen umgeben sind, nicht bekannt. Die Gotik bildet den einzigen Stil vor dem Bauhaus, der alle Bezüge zur Antike aufgegeben hat. Antike Formen lebten unmittelbar bis in die Romanik fort, seit der Renaissance wurde die Antike absichtlich wiederbelebt. Auch sonst fällt bei einem bündigen Überblick mehr auf, wie die gotische Kathedrale aus der Tradition herausfällt, als wo einzelne Elemente ansatzweise in der Romanik vorgebildet sind oder lokale Formen sich fortsetzen.

Griechischer Tempel und gotische Kathedrale haben die erhebende Wirkung gemein. Sie erscheinen dem Alltäglichen entrückt. Die äußere Form überwiegt offensichtlich den praktischen Nutzen. Disposition und Gliederung sind mit höchster Stringenz nach ihrer eigenen formalen Gesetzmäßigkeit durchgeführt. Beide erinnern geradezu an Architekturphantasien, der griechische Tempel durch seine Marmorkolonnaden, die nur dazu dienen, Eindruck zu machen, die gotische Kathedrale, weil ihre Wände so weit aufgelöst sind, dass sie, wie man 
oft gesagt hat, geradezu wie ein gläserner Schrein erscheint, dessen Bildfenster wie Edelsteine glänzen. Aber der griechische Tempel wirkt klar und übersichtlich in Disposition und Tektonik, gravitätisch in sich ruhend durch die ausgewogenen Proportionen und natürlichmenschlich durch das einfache Verhältnis von Tragen und Lasten, durch das Anspielen auf das Handwerk mit Elementen des Holzbaus und durch den Bezug der Säulen auf Menschenmaß. Die gotische Kathedrale wirkt dagegen unwirklich, wunderbar, übernatürlich durch die komplexe Disposition, die verwirrende Vielfalt der Glieder am Außenbau, die unüberschaubare Fülle von Statuen und Dekor an den Fassaden, im Innern durch die Dominanz der leuchtenden Glasfenster mit ihren zahllosen, vielfach vom weiten kaum erkennbaren Bildern und durch die filigrane Architektur, die übermäßig labil erscheint, weil verborgen ist, was sie zusammenhält. Sie wirkt dynamisch durch ihre Tiefenflucht auf den Chor zu, der als Zentrum hervortritt, und mehr noch durch ihr Streben in die Höhe, das einzelne Elemente wie besonders Spitzbogen und Fiale prägt und über Pfeiler und Wände hinaus sogar die Gewölbe mit ihren steil aufsteigenden Kappen ergreift, so dass der Kontrast zwischen tragenden Stützen und lastender Decke aufgelöst ist. Bei der Gotik treten keine Bezüge auf den Menschen in Erscheinung, alles ist maßlos in dem Sinn, dass keine auf den Menschen bezogenen Maßverhältnisse zur Anschauung kommen. Die Gliederung dient allein dazu, die Elemente des Raums zu rahmen und seine Dynamik zu unterstreichen. Im Innern herrscht eine abgezirkelte Ordnung, die so strikt abstrakten Richtlinien folgt, dass man sie oft als Stein gewordene Scholastik anspricht. Sie ist aber so komplex, dass ein normaler Betrachter sie kaum durchschauen kann. Die übernatürliche Wirkung, die allumfassende Ordnung und die Präsenz des Heiligen lassen die Kathedrale als ideale Glaubenssphäre, gewissermaßen als Himmel auf Erden erscheinen. Der gläserne Schrein weckt Assoziationen an das Himmlische Jerusalem, so wie es in der Bibel beschrieben ist.

Ein derartiger Abriss setzt ohne weiteres voraus, dass es über einzelne Beispiele hinaus eine allgemeine Vorstellung davon gibt, was der gotische oder klassisch griechische Bau ist, dass uns das Ideal an sich vor Augen steht. So etwas nimmt man bei anderen Stilen nicht an: Die Renaissance oder die Romanik kennen keinen Bautyp, der sie so selbstverständlich repräsentieren würde wie ,die Kathedrale“ die Gotik. Die Begriffe „Gotik“ und „Kathedrale“ werden oft sogar synonym gebraucht.

Bei einem kursorischen Überblick über den Zeitraum vom frühen 12. bis zum späten 15. Jahrhundert zeichnet sich freilich keineswegs ein einheitlicher gotischer Bautyp ab. Wie bei anderen Stilen herrscht je nach Zeit, Region und Funktion eine breite Vielfalt. Von Anfang an gab es zwei unterschiedliche Strömungen. Die eine ging von Paris und der nahe gelegenen Abtei St-Denis, der königlichen Grablege, aus. Sie erfasste städtische, fürstliche und bischöfliche Kirchen. Sie zeichnet sich durch großen Aufwand sowohl der Struktur als auch der festen Ausstattung und durch die komplexe Gestaltung aus. Abt Suger von St-Denis hat von der Schwindel erregenden Tektonik seiner neuen Kirche und der Pracht ihrer bunten Fenster geschwärmt. Die andere Richtung war bescheidener. Sie ging von Clairvaux in der Champagne aus und wurde durch die Zisterzienser im ganzen Abendland verbreitet. Die Bettelorden, Franziskaner und Dominikaner, die sich im 13. Jahrhundert verbreiteten, forderten noch mehr Einfachheit. Realiter sind freilich nicht einmal diese beiden Strömungen strikt voneinander getrennt. In Italien gleichen die Hauptkirchen der Bettelorden in der Disposition oft den Metropolitankirchen, und der so genannte Altenberger Dom im Bergischen Land ( $\mathrm{ab}$ 1255) übernimmt weitgehend die Gestalt einer hochgotischen Kathedrale nach französischem Vorbild, obwohl ihn Zisterzienser als Klosterkirche bauten.

Die Entwicklung der gotischen Architektur setzte um 1140 ein. Einzelne Elemente wie besonders Spitzbogen und Rippengewölbe kamen schon etwas früher auf. Während des folgenden Jahrhunderts entstand eine große Anzahl von höchst aufwendigen Kirchen im fran- 
zösischen Kronland; dort wurden alle Bischofskirchen erneuert. Trotz vieler individueller Lösungen strebte der Stil der neuen Kathedralen kontinuierlich in eine Richtung: zu immer filigraneren Strukturen, zur Öffnung der Wände in Fenster, zu schlankeren Proportionen und zu konsequenterer Ordnung. Die Mönchsorden blieben gewöhnlich bei einfacheren Strukturen. Von Frankreich strahlte die Gotik nach Europa aus, zuerst nach England, dann nach Spanien, schließlich in die deutschen Lande und nach Italien.

Trotz des nachhaltigen französischen Einflusses gingen die Länder aber ihre eigenen Wege. Lokale Traditionen lebten fort, und originäre Formen kamen auf. In England blieben Elemente der normannischen Kirchen im Innenraum gewahrt; für die Fassaden wurden höchst unterschiedliche Gestaltungen eingeführt; der Aufwand der Disposition wurde durch ein doppeltes Querhaus und einen mächtigen Vierungsturm gesteigert, während der komplexe Umgangschor, der für den Eindruck der französischen Kathedralen wesentlich ist, gewöhnlich nicht aufgenommen wurde. Die einheitliche Wirkung der franżösischen Kathedralen trat hier gegenüber der Vielfalt der Disposition und des Dekors zurück.

Als die umfassenden Bauaktivitäten im französischen Kronland zum Abschluss gekommen waren, rückten die individuellen Formen der verschiedenen europäischen Regionen in den Vordergrund. Die Vielfalt, die sich im Lauf der Spätgotik entwickelte, ist viel zu groß, als dass sie hier anschaulich wiedergegeben werden könnte. Beim Vergleich der Nachfolge des typisch französischen Stils, des decorated und perpendicular style in England, den überreich dekorierten Prachtbauten der iberischen Halbinsel oder der schlichten Backsteingotik in Norddeutschland und Skandinavien ergeben sich wohl mehr Unterschiede als Gemeinsamkeiten. Beim Dom von Florenz ( $\mathrm{ab}$ 1296) beispielsweise sind Bauformen von Bettelorden in gewaltige Dimensionen übersetzt, aber die Marmorinkrustation am Außenbau knüpft an die toskanische Protorenaissance an, eine noch stark von der Antike geprägte Richtung der Romanik. Ostpartie und Vierung haben der Renaissance mehr gegeben, als sie von der Gotik genommen haben. Die Kuppel, die in der Mitte des 14. Jahrhunderts geplant wurde, unterscheidet sich durch ihre typisch gotischen Elemente - hohe Steilung und Rippen - von den romanischen Vorgängern, aber sie bildet ein Unikum in der Gotik. Andererseits wurde sie zum Prototyp all der Kuppeln, die ein typisches Merkmal barocker Architektur bilden.

In dem breiten Spektrum der Sonderformen fällt eine ins Auge, die besonders in den deutschen Landen und vielfach auch auf der Iberischen Halbinsel seit Beginn der Gotik verbreitet war: die Hallenkirche (das bedeutendste von den seltenen Beispielen der Hochgotik: die Elisabethkirche in Marburg, ab 1235; eines von den zahllosen prachtvollen Beispielen der Spätgotik: die Annenkirche in Annaberg, Sachsen, ab 1499). Die Hallenkirchen wirken völlig anders als die hochgotischen Kathedralen: sie sind klar, hell und maßvoll. Im Unterschied zur Basilika sind hier alle Schiffe gleich hoch; statt vom Obergaden kommt das Licht aus den Fenstern der Seitenschiffe. In der Zeit, als sich die Hallenkirchen ausbreiteten, waren ihre Glasscheiben viel heller als in der Hochgotik. Gewöhnlich stehen die Pfeiler in so weiten Abständen, dass der gesamte Raum überschaubar ist. Die tektonischen Verhältnisse treten in Erscheinung, weil die Wände nicht so weit reduziert sind wie in der Hochgotik. Die Gewölbe sind nicht mehr in hochstrebende Kappen aufgelöst, sondern bilden eine einheitlich bauchige oder gewellte Decke. Dekor und Gliederung konzentrieren sich auf die Gewölbe, im Übrigen sind sie weitgehend eliminiert. Die Pfeiler sind rund oder polygonal, ohne Dienste, oft sogar ohne Kapitelle.

Die große Gruppe der majestätischen Kathedralen, die um die Wende vom 12. zum 13. Jahrhundert im französischen Kronland und in den angrenzenden Regionen entstanden, ist weitgehend repräsentativ für unsere Vorstellungen von der Gotik. Am nächsten kommt dem Ideal wohl der Kölner Dom. Dort wurde die neueste Form von Gotik aufgenommen, sogar noch weiter im Sinn der französischen Entwicklung ausgebildet und schließlich mit 
der einzigartigen Fassade verbunden, an der sich alles zum machtvollen Streben in die Höhe vereint. Schon im Verlauf der Gotik galten die Kathedralen des französischen Kronlandes als vorbildlich. Es ist mehrfach belegt, dass Architekten zur Planung von Neubauten zu ihnen geschickt wurden. Noch im 15. Jahrhundert ahmte der Innenraum der Pfarrkirche von L'Epine ganz auffällig die Kathedrale ihrer Diözese Reims nach. Bis hin nach Skandinavien richteten sich königliche oder fürstliche Kirchen nach dem Vorbild des französischen Kronlandes. Die schwedische Krönungskathedrale von Uppsala oder Westminster Abbey, die Grablege der englischen Könige, bilden bekannte Beispiele dafür. Auch der Altenberger Dom als Grablege der Herzöge von Berg gehört in diesen Bereich.

Die Prominenz der Gotik vor allen anderen Stilen als europäischer „Erinnerungsort“ passt eigentlich nicht recht zu unserem geistesgeschichtlichen Rückblick. Es besteht ein consensus omnium darüber, dass die Neuzeit nach dem Mittelalter mit der Renaissance begann. Seit den ersten Anfängen der Renaissance behaupteten die Avantgardisten vehement, dass vom Untergang des Römischen Reichs bis zu ihrer Zeit Ignoranz, Unwissenheit, blinder Gaube und Scharlatanerie geherrscht habe. Sie riefen dazu auf, aus dieser Dunkelheit herauszutreten und wieder zum Licht der Vernunft zu streben. Mit erheblicher Energie verwirklichten sie diese Maxime. Die moderne Wissenschaft und die ganze Art des Denkens, die mit ihr verbunden ist, entstanden in der Renaissance. Wir nehmen ohne weiteres an, dass bei allen Differenzen eine direkte Linie von den bahnbrechenden Humanisten bis zur Gegenwart führt. Inzwischen wird die Vorstellung vom dunklen Mittelalter in Fachkreisen zwar relativiert. Trotzdem ist sie noch ebenso lebendig wie die Bewunderung für die Gotik. Davon zeugt etwa die Verfilmung von Umberto Ecos Roman „Der Name der Rose“. Victor Hugo lieferte 1831 in seinem Roman „Notre-Dame de Paris“ vielleicht ein Argument dafür, dass die Gotik heute als Inbegriff abendländischer Architektur erscheinen kann, obwohl uns die Innovationen, die zu Beginn der Neuzeit ihr Ende herbeiführten, näher stehen: Bis zur Erfindung des Buchdrucks durch Gutenberg, , ,der Mutter aller Revolutionen“, schreibt er, habe die Baukunst dem menschlichen Geist Dauer verliehen, dann habe der Buchdruck diese Aufgabe übernommen: „Das Buch tötete den Bau“.

Der geistige Umbruch vom Mittelalter zur Neuzeit prägte vier Jahrhunderte lang den Blick auf die Gotik oder beeinflusste ihn zumindest markant. Das generelle Verdikt über das Mittelalter galt weithin auch für sie bzw. für die gesamte mittelalterliche Architektur vom Untergang des Weströmischen Reichs bis zum Beginn der Renaissance. Lange wurde die mittelalterliche Architektur als eine Einheit hingestellt, ohne nachvollziehbar zwischen Stilepochen oder Entwicklungsstufen zu differenzieren. Die Avantgardisten warfen ihr vor allem vor, dass sie nicht der Antike folgte, weil diese als Lehrmeister beim Aufbruch in die Neuzeit galt. Daher nannte man die mittelalterliche Architektur, auch nachdem sie längst durch die Renaissance zurückgedrängt war, „,modern“. „Moderne“ Architektur bedeutete in der Renaissance gewöhnlich veraltete, überholte, primitive Architektur. Man stellte sich vor, die „moderne“ Bauweise sei während der Völkerwanderung von den „barbarischen“ Stämmen, speziell den Goten, ins Abendland eingeschleppt worden. Trotzdem nannte man sie damals noch nicht ,gotisch“. Die Deutschen standen im Ruf, sie zu ihrer typischen Art entwickelt und im Lauf des Mittelalters etwas verbessert, wenn nicht sogar erfunden zu haben. Daher bezeichneten die italienischen Avantgardisten seit dem Beginn der Renaissance die mittelalterliche Bauweise auch als „deutsche Manier" (maniera tedesca). Im Hochmittelalter wurde die gotische Bauweise meist als opus francigenum, also als französisch oder fränkisch angesprochen.

Die Orientierung an den großen geistigen Leistungen des Altertums hatte ihren guten Sinn und erwies sich als sehr effizient, aber die Festlegung der antiken Architektur als Norm war letztlich willkürlich. Die Avantgardisten kommentierten die mittelalterliche Architektur 
aus dieser Warte voreingenommen und polemisch, unlogisch und widersprüchlich. Hässlich und geistlos sei sie, hieß es, ihr fehle jedes vernünftige Maß, ihre Glieder seien beängstigend schmächtig, sie ertrinke in wirrem Flitter. Nur vage geben die Schmähungen zu erkennen, welche Art von Bauten gemeint war und welche Züge konkret abgelehnt wurden: Die Merkmale, die sich abzeichnen, sind für die Gotik typisch. Aber sie wurden pauschal auch auf die romanischen Bauten übertragen.

Zudem brachten die Reformatoren Kritik an der Gotik auf. Martin Luther nannte so aufwendige Kirchen wie den Kölner Dom oder das Ulmer Münster „unpraktisch“ (1538). Kirchen sollten seiner Meinung nach hauptsächlich für Predigten dienen. Sie sollten übersichtlich sein und den Gläubigen ermöglichen, Gottes Wort akustisch gut zu verstehen. Seitenschiffe, übertriebene Dimensionen und große Höhe erschienen ihm daher eher hinderlich.

Die an der Antike orientierte Polemik gegen die gotische Architektur kam in Mittelitalien auf und breitete sich von dort, wie so viele neue Ideen der Renaissance, während des 16. Jahrhunderts in ganz Europa aus. Sie wurde allenthalben verbindlich für die Avantgardisten, aber es gab Einschränkungen. Selbst Fundamentalisten konzedierten, wenn auch abschätzig, gotische Bauten könnten, so kunstlos sie seien, auf Ungebildete immerhin gefällig wirken. Schon in Norditalien wurde die Polemik nicht so apodiktisch und pauschal wie in Florenz vorgetragen. Während die Gotik in Italien als Fremdimport erschien, blieb in anderen Ländern stets in Erinnerung, dass sie zum eigenen Erbe gehörte. Sie trat zu dominant in Erscheinung, um das vergessen zu lassen. Als die Renaissance begann, waren besonders in Deutschland und Spanien viele gotische Kirchen im Bau oder noch nicht vollendet. In Frankreich war man noch viel später bemüht, die Gotik nachzuahmen, um die schweren Schäden an den alten Kirchen zu restaurieren, die durch die Hugenottenkriege (ab 1562) entstanden waren.

In die lokale Literatur zu einzelnen gotischen Bauten ging das Verdikt über die Gotik meist nicht ein. Der Stolz auf das eigene Wahrzeichen überwog die theoretische Distanzierung. Das gilt sogar für Florenz. Albrecht Dürer stellt in einer Abhandlung über Architektur fest, die Maßregeln der Antike seien zwar wichtig, aber auch sie seien nur von Menschen ausgegeben worden, und deshalb könne man jetzt ebenso wie damals Neues erfinden. Die Illustrationen $\mathrm{zu}$ dem Text verbinden antike und gotische Formen miteinander. Der französische Architekt Philibert de l'Orme übernimmt in seinem Architekturtraktat (1567) die neuen antikischen Normen, aber er stellt ihnen die eigenständigen Leistungen der französischen Gotik gegenüber, besonders die Konstruktion von Gewölben und den Steinschnitt. Nicht selten wurde, ziemlich unabhängig davon, wie stimmig das ist, einfach behauptet, die antiken Regeln passten auf gotische Kathedralen - so hieß es in Chartres, Mailand, Köln oder Straßburg.

Am leichtesten ließen sich die gotischen Bauten gegen den Vorwurf verteidigen, sie wären nicht systematisch proportioniert. Soweit der Vorwurf nur darauf abzielte, dass die Maßverhältnisse nicht so ausgewogen wie bei antiken Bauten seien, trifft er ja wenigstens für die hochgotischen Kathedralen zu. In seiner üblichen pauschalen Form ging er aber an der Sache vorbei. Die gotischen Kirchen waren nicht weniger konsequent nach präzisen Proportionen geplant wie die Bauten der Antike und der Renaissance. Dafür gibt es viele Zeugnisse; für die Spätgotik das schlagkräftigste ist wohl die systematische Beschreibung des oberrheinischen Architekten Lorenz Lechler, wie eine Kirche gestaltet werden soll. Einfache und klare Proportionen bilden da die Leitlinie. Am berühmtesten wurde die Idee des Gabriele Stornalocco aus Piacenza, den Aufriss des Mailänder Doms nach einem gleichseitigen Dreieck zu bemessen (1391). Sie wurde 1521 in einem Kommentar zu dem einzigen erhaltenen Architekturtraktat aus der Antike aufwendig publiziert und noch weiter ausgesponnen; die deutsche und französische Architekturtheorie behandelte Stornaloccos Idee bis ins späte 17. Jahrhundert 
hinein als mustergültig. 1589 entfachten die Bürger in Bologna einen wahren Sturm, damit die gotische Kirche von San Petronio wie der Mailänder Dom nach den Maßen eines gleichseitigen Dreiecks vollendet werde, weil sie angeblich so begonnen wurde. Die wissenschaftliche Kunstbetrachtung des 20. Jahrhunderts verallgemeinerte Stornaloccos Idee. Generell sollen demnach die Pläne der Gotik auf geometrischen Figuren basieren, während diejenigen der Renaissance arithmetisch ausgerichtet seien. So ergibt sich eine perfekte Übereinstimmung mit den üblichen Vorstellungen von den Epochen: Die Renaissance hielt sich an rationale, die Gotik an irrationale Maßverhältnisse. Bewiesen ist die These nicht, wirklich plausibel auch nicht, aber weit verbreitet.

Auch unabhängig von der Theorie lebte die Gotik lange weiter, weit über eine Übergangszeit oder über zurückgebliebene Regionen hinaus. Bei den Arbeiten an unvollendeten oder beschädigten gotischen Bauten wurde oft die „moderne Manier“ imitiert, um die Einheit des Stils zu wahren. So wurde etwa bei der Wiederherstellung der Kathedrale von Orléans nach der Zerstörung durch die Hugenotten auch gleich das romanische Querhaus, das noch erhalten war, dem Rest des Baus angepasst. Vielfach kam Traditionsbewusstsein durch das Festhalten am gotischen Stil zum Ausdruck, die Universitätsbauten von Oxford und Cambridge liefern Beispiele dafür. Den Anschluss an die einheimische katholische Tradition sollte offenbar die Jesuitenkirche St. Mariae Himmelfahrt in Köln demonstrieren, die 1618-1678 in rein gotischen Formen mit Türmen im romanischen Stil errichtet wurde. Anscheinend war die Gotik von der Aura des Sakralen umgeben. Das wirkte sich in Frankreich im 16. Jahrhundert so aus, dass an vielen Schlössern die Kapelle gotisch blieb, während das Haus sonst den neuen Stil der Renaissance aufnahm.

Vor allem bestand die Gotik nördlich der Alpen in der Art fort, dass Neubauten ihre Disposition und besondere Art von Tektonik bewahrten und nur oberflächlich mit Dekor der Renaissance ummantelt wurden. Die filigrane Konstruktion lebte in neuem Kleid weiter. Die Pfarrkirche von St-Eustache in Paris, die auf Initiative König Franz' I. erneuert wurde (ab 1532), bildet das markanteste Beispiel für die Verbindung von Historismus und neuer Mode. Der gotische Dekor ist ersetzt durch Elemente aus dem Repertoire der Renaissance. Aber dieser neumodische Überzug ist nicht im Stil der Renaissance versetzt. An Stelle der Dienste etwa stehen lauter Säulchen oder Pilaster übereinander. Alles ist innen wie außen gotisch disponiert, mit steilen, hochstrebenden Räumen, Auflösung der Wände, außen mit Streben und hohem Dach. Schon 1479-1483 ließ der französische Kardinal Guillaume d'Estouteville in Rom eine Kirche mit ähnlichen Merkmalen errichten, S. Agostino, offensichtlich in der Absicht, vor aller Welt zu demonstrieren, wie die gute Architektur auf französische Weise aussieht. Die deutsche Nationalkirche in der Ewigen Stadt, S. Maria dell'Anima, wurde ausdrücklich „auf deutsche Manier“, wie es 1499 in den Bauakten heißt, errichtet, mit der Disposition einer gotischen Hallenkirche und der Gotik angepasstem Renaissance-Dekor. Ein typisches Beispiel für dasselbe Phänomen bildet der erste große Neubau einer lutherischen Kirche: die Marienkirche in Wolfenbüttel (ab 1608).

Als Frankreich unter dem Sonnenkönig gegen Ende des 17. Jahrhunderts die kulturelle Führung in Europa übernommen hatte, wandelte sich allmählich der Rückblick auf die Gotik. In Frankreich wuchs der Stolz auf die eigenen majestätischen Monumente. Die Ste-Chapelle spiegelte für Viele den Glanz Ludwigs des Heiligen. Die zentralistische Bündelung der geistigen Kräfte in der 1671 gegründeten Académie Royale d'Architecture trug wesentlich dazu bei, den stilistischen Charakterzügen und dem Geschichtsbild der Gotik endlich deutlichere Konturen zu verleihen. Dem Architekturtheoretiker Jean-François Félibien gelang es 1687, mehrere Stufen in der mittelalterlichen Architektur zu unterscheiden; er setzte die Gotik als eigenständigen Stil gegen die Romanik ab. Der Begriff „Romanik“ wurde allerdings erst zu Beginn des 19. Jahrhunderts eingeführt. 1699 zeigte Félibien auch eine Entwicklung inner- 
halb der Gotik auf, von der glanzvollen Epoche der Hochgotik, die er in der Zeit Ludwigs des Heiligen ansetzte, zur flamboyanten Spätgotik, die er als Niedergang wertete, als Absturz in eine konfuse Massierung von überbordendem Ornament und in übermäßige Finesse.

1774 unterschied Louis Avril erstmals nationale Eigenheiten in der Gotik: in Frankreich und England sei sie klar in den Grundformen, in Italien bleibe sie der Antike näher, in den deutschen Landen und im Norden sei sie reich an Ornament, in Spanien habe sie auf Grund des maurischen Einflusses exzessive Finesse angenommen. Jetzt erhob sich auch die Frage, wie die mittelalterliche Bauweise wirklich aufgekommen sei. Franzosen und Engländer hatten die Bezeichnung ,, deutsche Manier" nie verwendet. Sie führten unter Bezug auf die Goten den Begriff "gotisch“ dafür ein. Allerdings wurde zunehmend klar, dass die Goten ebensowenig wie Deutsche oder Germanen als Erfinder in Frage kamen, weil sie zu primitiv waren. Im 18. Jahrhundert verbreitete sich die Ansicht, Araber, Sarazenen oder Mauren hätten die mittelalterliche Bauweise ins Abendland eingeschleppt, als sie, wie es der englische Literat John Evelyn 1707 formulierte, die ,zivilisierte Welt“ vom Süden her überrannten, kurz nachdem die wilden Stämme von Goten und Vandalen vom Norden her eingedrungen seien. Im späteren 18. Jahrhundert wurde der Begriff „gotisch“ im heutigen Sinn auf die spätere mittelalterliche Architektur eingeschränkt. Gleichzeitig wurde die sarazenische Erfindung des Stils auf sie übertragen mit der Variante, die Kreuzfahrer hätten die Gotik aus dem vorderen Orient mitgebracht.

Die vom Vorbild der Antike abgeleiteten Normen, die den Blick auf die Gotik bisher in der Theorie verstellt hatten, verloren in Frankreich allmählich an Gewicht. Von dem konventionellen Verdikt über die Gotik blieb zunächst noch erhalten, dass ihr Dekor weiterhin in Bausch und Bogen abgelehnt wurde. Zum Dekor zählten im weiteren Sinn auch die bunten Fensterscheiben; sie wurden oft durch farbloses Glas ersetzt. Das Bild von der reinen Gotik in strahlendem Licht, dem sich die hellen Fenster so weit wie irgend möglich öffnen, hat seit dem französischen Klassizismus Bestand. Zudem setzte damals die Entwicklung ein, alte Kirchen freizulegen, von ihren ursprünglichen Anbauten zu „befreien“, so dass sie möglichst wenig eingeschränkt allseits gut sichtbar sind.

Der Geltungsbereich der antikischen Normen wurde seitdem offen eingeschränkt: Nur noch für den Dekor sollten sie verbindlich sein. Die substantielle Struktur der gotischen Bauten, ihre aus italienischer Warte so oft kritisierte filigrane Konstruktion, die légèreté und hardiesse (Leichtigkeit und Kühnheit), wie man in Frankreich sagte, und die Wirkung, die sie hervorbrachte, fanden zunehmend Bewunderung. Um 1740 hieß es in Frankreich, die Wiedergeburt der guten Architektur, die mit dem Römischen Reich untergegangen sei, habe trotz aller Unterschiede zur Antike eigentlich mit der Gotik eingesetzt. Weil sie die wahren tektonischen Verhältnisse zum Ausdruck gebracht habe, wurde die Gotik, obwohl sie Kritik hervorrief, jetzt oft der neuen Architektur in der Nachfolge der Renaissance vorgezogen.

Die theoretische Neubewertung der Gotik setzte an einer Schwachstelle der klassischen Architekturtheorie an. Der Vorrang der Säulen wurde konventionell mit den klaren Verhältnissen von Tragen und Lasten begründet, die an antiken Portiken mit freistehenden Säulen herrschen. Aber realiter stellte man inzwischen Säulen gewöhnlich nur als Blendgliederung an Wände, die sich in Arkaden öffnen und Gewölbe tragen. Die französischen Theoretiker setzten nun die typisch gotische Struktur wegen ihrer légèreté in Parallele zu der des griechischen Tempels. Die Pfeiler, die als allein tragende Elemente der aufgelösten Wände übrig bleiben, wurden den Säulen gleichgestellt. Diese Idee wurde sogar auf gotische Türme übertragen, wenn sie so luftig sind wie derjenige des Straßburger Münsters und keine geschlossenen Wände aufweisen. Die ideale Architektur sollte griechische Form, also Säulen und Gebälk, mit dem Tragen von Gewölben verbinden, und das wurde in Parallele zur gotischen Tektonik gesetzt. Die Theorie mag etwas trocken klingen, aber sie wurde wesent- 
lich für die französische Architektur. Ihr Stil wird "graeco-gotisch“ genannt. Wieder einmal erscheint aus dieser Warte die Gotik in neuem Gewand.

Wie eng der französische Klassizismus, der unter dem Sonnenkönig einsetzte und von ihm gefördert wurde, mit der Rückbesinnung auf die Gotik verbunden war, zeigt am deutlichsten die Schlosskapelle von Versailles (ab 1699). Die Formen sind klassisch, ein Säulenportikus beherrscht die Erscheinung im Innern, und trotzdem sieht man, dass sich die Planung an der Ste-Chapelle orientierte, der unter Ludwig dem Heiligen errichteten Kapelle des königlichen Amtschlosses in Paris (Palais de la Cité). Seinen Höhepunkt erreichte der „graeco-gotische" Stil in dem grandiosen Raum des Pariser Pantheons (ab 1755) mit seinen hohen komplexen Gewölben und seiner Flut von Licht. Wenn hier, außer dem theoretischen Konstrukt von der wahren Tektonik und dem Ideal der Lichtfülle, etwas an die Gotik erinnert, dann ist es die erhebende Wirkung.

Auch in England nahm das Interesse an der Gotik im 18. Jahrhundert deutlich zu. Aber der Zugang zu ihr war viel unbefangener als bei den französischen Akademikern. „One must have taste to be sensible of the beauties of Grecian architecture, one only wants passions to feel Gothic", urteilte der Schriftsteller Horace Walpole. Hinter dem Interesse am Mittelalter stand hier weniger eine normative Ästhetik als ein gewisser Sinn für Skurrilität, den die Engländer damals auch in anderen Bereichen weidlich auslebten. Man goutierte die pittoresken Reize der Gotik, obwohl sie keineswegs kunstvoll erschien, sondern wie damals üblich kritisiert wurde.

Neuerdings wurde die Gotik im privaten Bereich imitiert. Horace Walpole machte diese Mode populär mit seinem Wohnhaus, Strawberry Hill, das er ab 1753 bei London baute. Alte Burgen wurden nun im ,ancient saxon-gothic style " restauriert. Künstliche Ruinen im gotischen Stil und sogar verfallene gotische Kirchen wurden in Landschaftsgärten integriert. Mit einiger Verzögerung wurde die von England ausgehende Neugotik auf dem Kontinent rezipiert, speziell in Deutschland. Ein frühes Beispiel dafür bildet das sog. Gotische Haus in Wörlitz (ab 1773).

Die englische Neugotik lebte von der Lust am „dunklen Mittelalter“. Für Walpole bildete der "phantastische Bau“ seines Hauses, wie er selbst kommentierte, die Szene, die den Autor des "Castle of Otranto“ inspirieren sollte. Der Roman erzählt eine düstere, geheimnisumwitterte Geistergeschichte, die im Mittelalter spielt. Walpole leitete mit ihm das literarische Genre des Schauerromans ein, das ungeheuer populär wurde. Die Kommentare zum Gotischen Haus in Wörlitz fielen dementsprechend aus. Auch wenn der Bau als gelungen gerühmt wurde, galt sein Stil allgemein als ,geschmacklos“. Gefallen erregte, dass hier die Vorstellung von der „,barbarischen“ Zeit des Mittelalters evoziert wurde. Das Gotische Haus, fand ein Besucher, trage "ganz das Gepräge jener Jahrhunderte des Aberglaubens, der Zwietracht und der Galanterie". Auch schauerliche Geschichten aus dem Mittelalter, wie sie etwa das Nibelungenlied oder Shakespeare in seinen Königsdramen wiedergeben, übten damals große Attraktion aus.

Die gotischen Bauten selbst erschienen in einem solchen geistigen Umfeld als Zeugnisse des dunklen Mittelalters. Als der dänische Schriftsteller Jens Baggesen 1791 ins Straßburger Münster kam, wurde er von wahrem Ekel vor dem Aberglauben erfasst: „Zitternd betet man darin an. Die ganze Physiognomie ist die des Catholizism: Aberglaube zeigt sich in jeder Verzierung. Das Papsttum mit allen Emblemen der Mönchsherrschaft ist auf seinen Mauern abgedruckt. Aus diesem Gesichtspunkte betrachtet, ist es eine Hierarchie von Stein und Eisen, die das Auge mit tausend phantastischen Spielwerken blendet“. In der Zeit der Französischen Revolution wüteten die radikalen Vertreter der Aufklärung gegen die „Embleme der Mönchsherrschaft“ und Denkmäler des „Fanatismus“. Viele mittelalterliche Kirchen wurden zerstört, geschändet und entweiht. Die Denkmäler des „Fanatismus“ wurden zu „Tempeln 
der Vernunft“ umgewandelt. Victor Hugo schrieb dazu: „Dann haben die religiösen und politischen Aufstände die Menschen blind und rasend gemacht, und die also verblendeten haben sich über die Bauten gestürzt, haben [...] die Statuen abgeschlagen, das eine Mal, weil sie einen Bischofshut, ein anderes Mal, weil sie eine Königskrone trugen“.

Die Verbindung von allen dunklen Seiten des Mittelalters mit der Gotik lebt bis heute in dem englischen Epitheton gothic fort, das unter anderem die populären Schauerromane bezeichnet und inzwischen auch in die populäre neudeutsche Sprache eingegangen ist. Gothic bezieht sich in diesem Sinn nicht allein auf einen historischen Stil, sondern bedeutet allgemein skurril, schrill, grob, primitiv und schauerlich.

Allmählich bahnte sich im 18. Jahrhundert allerdings rückhaltlose Bewunderung für die Gotik an. Die Grundlage für den Wandel bildete die generell neue Bewertung des Primitiven, der Jean-Jacques Rousseau in seinem berühmten ,Diskurs über die Ungleichheit“ (1754) nachhaltig Ausdruck verliehen hat. Hier erscheint das Primitive, weil es der menschlichen Natur nahe steht, weit erstrebenswerter als die künstliche Perfektion der modernen Zivilisation, weil sie gegen den ursprünglichen Drang nach Freiheit unter Gesetze gezwungen ist. Diese Sicht bezog sich auf alle Seiten der Gesellschaft; auch bildende Kunst und Literatur waren davon betroffen. Um Freiheitsdrang kreisen die typischen Werke des „Sturm und Drang“, die oft im mittelalterlichen Milieu spielen.

Ausgehend von England, sprengte die Kunsttheorie die Fesseln der normativen Ästhetik. Individuelle Einbildungskraft und Affekte ersetzten das Korsett akademischer Regeln. „Regeln sind wie Krücken, eine nothwendige Hülfe für den Lahmen, aber ein Hindernis für den Gesunden", urteilt Lessing in der "Hamburgischen Dramaturgie“. Mit Regeln schaffe man nur Gewöhnliches, das wahre Genie steige zum Erhabenen auf, indem es seine natürliche gestalterische Kraft auslebt. Shakespeare, der in seinen Dramen sämtliche klassischen Regeln verletzt hat und trotzdem der bühnenwirksamste Autor ist, bildete ein Paradigma für das Genie. Kunstwerke sollten nicht mehr in erster Linie mit der Vernunft betrachtet werden, wie es die klassische Architekturtheorie seit der Renaissance forderte. Bisher hieß es, das Unüberschaubare, Verwirrende, Erstaunliche gehöre nicht zu den Kriterien der wahren Kunst. Nur das gemeine Volk lasse sich von seinen Sinnen verführen. Jetzt fand die Wirkung auf die Sinne Bewunderung. Das Erhabene wurde gesucht, der Effekt, der so grandios, so überraschend und unfasslich ist, dass er den Verstand überwältigt und die Gefühle aufwühlt.

Die neue Haltung beeinflusste sogar die französischen Akademiker, auch wenn sie den Normen treu blieben und daher den gotischen Dekor ablehnten. Etienne-Louis Boullée schreibt, obwohl die Goten noch nicht gewusst hätten, wie gute Architektur sein soll, hätten sie vermocht, ihren Kirchen „une grande charactère“ zu verleihen. Sie hätten „die Magie in die Kunst eingeführt“, denn da sie die Tektonik verborgen hätten, wirkten ihre himmelstürmenden Kirchen wie von überirdischen Mächten zusammengehalten. Die Räume erschienen weit größer, als sie sind, schrieb der Architekturtheoretiker Jacques-François Blondel um 1760, sie hätten einen religiösen Charakter und evozierten Frömmigkeit. Boullée begründete das Phänomen, dass die Goten solche Werke schaffen konnten, ohne die richtigen Regeln zu kennen, mit ihrer Primitivität, damit, dass sie „den Eingebungen ihres Genies folgten, denn der Mensch ist immer etwas, wenn er nur die ihm von der Natur gegebenen Möglichkeiten ausnützt, während der Mensch, der nur nachäfft, verdirbt und überhaupt nichts ist".

Der junge Goethe wagte 1773 in einem Essay über das Straßburger Münster und seinen Baumeister Erwin von Steinbach den einschneidenden Schritt, alle Vorbehalte gegen die Gotik auszuräumen. Die Schrift nimmt Rücksicht auf die klassische französische Architekturtheorie, aber sie ist mitreißend - und hinter ihr steht die Haltung Rousseaus. Im Sinn der neuen Begeisterung für das Primitive ist die Reserve gegen die Gotik geradezu in ihr Ge- 
genteil verkehrt. Goethe stimmt einen flammenden Hymnus auf die erhebende Erscheinung des Baus und das Genie seines Schöpfers an. Er ruft die Deutschen dazu auf, die Gotik als ihr eigentliches künstlerisches Erbe zu begreifen. Dieser Anspruch war an sich nicht überraschend. Die Wortführer der Renaissance verbanden die Gotikja seit jeher mit den Deutschen, und auch in Frankreich oder England wuchs mit zunehmendem Verständnis für die Gotik die Neigung, sie als nationales Erzeugnis anzusehen. Das Besondere besteht darin, dass der Hymnus von der Anziehungskraft des Primitiven geprägt ist. Die Deutschen erschienen in der Romania gewöhnlich als unkultiviert, als „Barbaren“, wie man sie oft abschätzig nannte, nicht viel besser als ihre germanischen Vorfahren, die für den Untergang der antiken Kultur verantwortlich gemacht wurden. Die Verbindung der Gotik mit den Deutschen erwächst bei Goethe aus der Parallele zwischen dem Volk, „das alle Welt barbarisch nennt", und der Bauweise, die aus der Warte der Klassik ebenso abgeurteilt wurde: „tretet hin, und erkennt das tiefste Gefühl von Wahrheit und Schönheit der Verhältnisse, wirkend aus starker, rauher, deutscher Seele, auf dem eingeschränkten düstern Pfaffenschauplatz des medii aevi“‘

Goethes Essay traf den Nerv der Zeit. In der deutschen Romantik erwachte eine wahre Begeisterung für die Gotik. Sie fand ihren Ausdruck in zahllosen Schriften und in Bildern, im Sammeln gotischer Werke und in Forschungen, in neuen Bauten im gotischen Stil und, für die Nachwelt am deutlichsten, darin, dass viele Kirchen, die unvollendet geblieben waren, nach Jahrhunderten nun fertig gestellt wurden. Die Erscheinung der deutschen Gotik ist wesentlich durch diese Baumaßnahmen geprägt. Viele der typischen hohen filigranen Türme, wie derjenige des Ulmer Münsters, entstanden erst im 19. Jahrhundert. Der Kölner Dom, das Musterbeispiel für unsere Vorstellungen von der Gotik, wurde zum großen Teil erst damals ausgeführt. Sulpiz Boisserée, der eine bedeutende Sammlung gotischer Bildwerke zusammentrug, hatte schon 1810 vorgeschlagen, ihn zu vollenden. Die Idee erhielt weitere Nahrung, als der Plan für die Westfassade gefunden wurde, 1842-1880 wurde der Vorschlag ausgeführt - unter dem Patronat eines Protestanten, des preußischen Königs Friedrich Wilhelm IV. Die Anziehungskraft der Gotik überwand bei derartigen Unternehmungen mehrfach die konfessionellen Grenzen.

Die Neubauten der deutschen Romantik im gotischen Stil waren von einem ganz anderen Geist getragen als die Neugotik, die von England ausgegangen war. Der gotische Stil wurde als besonders naturnah betrachtet und schien daher angemessen für die Deutschen, deren Einschätzung immer noch von der "Germania“ des Tacitus beeinflusst war: „mit der Ursprünglichkeit und Freiheit ihrer Natur" ein ,wahres Urvolk“ (Schinkel). Seit langem wurden die gotischen Elemente von den primitiven Behausungen der Germanen oder Gallier in den Wäldern abgeleitet. Diese historische Entwicklungstheorie wurde nun umgeformt zu stimmungsvollen Beschreibungen eines Eindrucks. So schreibt Friedrich Hegel, beim Betreten eines gotischen Doms sei man , an die Wölbungen eines Waldes erinnert, dessen Baumreihen ihre Zweige zueinander neigen und zusammen schließen“. Friedrich Schlegel bewunderte darüber hinaus das „organisch Wachstumshafte“ an der Gotik, das einem „unermesslichen Gebilde der kristallisierten Natur“ gleiche (1804/5). Er spürte darin die „innere Geometrie der Natur", auch wenn sie ,nicht mehr im einzelnen sichtbar hervortritt, sondern alles frei im unendlichen Leben blüht und seine Schönheit entfaltet“. Damit berührte er eine Metapher, die schon im Mittelalter verbreitet war, und die Theorie von der Proportionierung nach Stornaloccos gleichseitigem Dreieck.

Die deutschen Romantiker bewunderten wie zuvor die französischen Theoretiker die „Entmaterialisierung“ der Steinmassen und das Emporstreben in die Höhe. Aber sie haben diese Verhältnisse stimmungsvoll artikuliert und überdies einen höheren Sinn darauf gesetzt: „Jetzt ward der Geist völlig Sieger über die Masse oder Materie“ (Schinkel). Heinrich Heine bewunderte die Kraft einer Epoche, ,die selbst den Stein so zu bewältigen wusste, dass er 
fast gespenstisch durchgeistigt erscheint, dass sogar diese härteste Materie den christlichen Spiritualismus ausspricht" (1836). Caspar David Friedrich hat die romantische Vision von der Gotik als Inbegriff christlicher Frömmigkeit mehrfach in Bildern wiedergegeben.

„Die Tempel blieben dem Auge heilig, als die Götter längst zum Gelächter dienten [...] Die Menschheit hat ihre Würde verloren, aber die Kunst hat sie gerettet und aufbewahrt in bedeutenden Steinen; die Wahrheit lebt in der Täuschung fort, und aus dem Nachbild wird das Urbild wieder hergestell"“, schrieb Friedrich Schiller in seinen Briefen „Über die ästhetische Erziehung des Menschen“ (1795). Ähnliches galt nun für die gotische Kathedrale. Sie entsprach dem damaligen Ideal des Kunstwerks, das den praktischen Nutzen hinter sich lässt, um wiederzugeben, was die Menschheit im Grunde bewegt: das Streben nach Gott oder der Natur oder nach Wahrheit und Selbsterkenntnis. Dadurch ragt das Kunstwerk aus dem alltäglichen Betrieb heraus und überdauert die Zeiten. An die Stelle der normativen Ästhetik trat die geistige Tiefe als Norm. Mit dieser Haltung verband sich, letztlich ähnlich wie bei Rousseau, ein kulturkritischer Aspekt. Im Mittelalter wie bei den alten Griechen sah man Kunst und Leben zu einer Einheit verbunden. Im modernen Leben dagegen schien die Kunst durch flüchtige Moden verdrängt. Caspar David Friedrich und Karl Blechem haben viele Bilder von Ruinen gotischer Kirchen gemalt, umgeben von eisigen Wäldern, abgestorbenen Bäumen, Friedhöfen und einsamen Denkern, die mit ihrer elegischen Stimmung Gedanken daran wecken, dass die wahre Kunst verfallen ist, weil das gesellschaftliche Leben seinen höheren Sinn verloren hat.

Die „Renaissance“ der Gotik wirkt seit der Romantik bis in unsere Zeit kontinuierlich fort, wenn auch beträchtlich gewandelt. Immer mehr Kirchen ahmten im Verlauf des 19. Jahrhunderts den gotischen Stil in Architektur und Ausstattung täuschend ähnlich nach. Daneben wurden auch alte gotische Kirchen restauriert und so hergerichtet, wie es der Vorstellung von Gotik entsprach. Ein bezeichnendes Element für entsprechende Annäherungen an das Ideal bildet die Ergänzung von Strebebögen, wenn sie fehlten, wie etwa an den Domen von Mailand, Salisbury und Regensburg. Die angefügten Strebebögen haben keine tektonische Funktion; sie sind eher Embleme für Gotik. Die Neugotik ebbte im frühen 20. Jahrhundert ab. Manchmal lebte sie dann noch für kurze Zeit in abstrakteren Formen fort, wie in Bruno Tauts Vorstellungen von einem ,Kristallpalast“. Immerhin sind heute noch zwei spektakuläre neugotische Dome im Bau: in Amerika die Kathedrale von New York, St. John the Divine (ab 1892), die eine der größten Kirchen der Welt werden soll und völlig stilrein bis heute bei gotischen Formen bleibt, und in Europa der 1882 von Antoni Gaudí begonnene Tempel der Sagrada Família in Barcelona, bei dem gotische Formen zunehmend in neuem Stil umgedeutet werden, weil es hier darauf ankommt, die überwältigende Wirkung der Gotik auch noch im Zeitalter des Massenkonsums zu erreichen.

Auch den Blick auf die Gotik hat die Romantik bis weit ins 20. Jahrhundert hinein und teilweise noch bis heute beeinflusst. Vielfach galt das einst als barbarisch verschriene Mittelalter nun zusammen mit der altgriechischen Klassik als ,Zeit der stärksten europäischen Kultur“, wie Bruno Taut gesagt hat. Georg Biermann bezeichnete das ,,dunkle Mittelalter“ als ,herrliche Epoche des Lichts, das die Völker der Erde im Geiste und in der Kunst zusammenführte“ (1918). Damals sah Oswald Spengler in den gotischen Domen den Ausdruck des abendländischen Weltgefühls. Der avantgardistische Städteplaner Ludwig Hilbersheimer teilte diese Auffassung: „Den höchsten künstlerischen Ausdruck fand Europa in der dem religiösen Bewusstsein des Mittelalters entspringenden Baukunst. Wobei unter Baukunst die Synthese von Architektur, Plastik und Malerei zu verstehen ist. Diese höchste Einheit verkörpert die Gotik in der Kathedrale: eine kristallisierte Idee von konsequenter Folgerichtigkeit. Alles irgendwie Dekorative unmöglich machend. Vehemente Entladung seelischer Spannungen. Grandioses phantastisches Sichauftürmen. Innigstes religiöses Gefühl vergöttlicht, entmaterialisiert 
die Materie. Lässt alle Schwere vergessen [...] Das sind Höhepunkte europäischen Kunstschaffens. Hervorgegangen aus der Einheit des Wollens und Tuns. Verbunden durch das zugrundeliegende verbindende religiöse Gemeinschaftsgefühl, das jedem Tun, auch dem geringsten, seinen Wert gab, zu einer gottesdienstlichen Handlung machte".

Ähnlich wie schon bei Goethe, war der Turm das Paradigma für die rein geistige Ausrichtung der Gotik. Bruno Taut und Walter Gropius haben ihn 1919 so angesprochen. Gropius: „Sehen Sie sich den Turm einer gotischen Kathedrale an. Ist er nicht völlig zwecklos? Ja er trägt vielleicht eine Glocke aber um für diese Ständer zu sein braucht es nicht der abertausend Figuren und Fialen und Sternblumen aus Stein. Ein solcher Turm war eben der Ausdruck einer seelischen Bewegung, eines religiösen Sehnsuchtsgefühls im ganzen Volke“. Die Kathedrale steigt in der Darstellung auf dem Bauhaus-Manifest geschlossen zu einem Turm auf. Joris-Karl Huysmans schreibt in dem Roman „La cathédrale“ (1898) über die Kathedrale von Chartres: Sie „reckte die beiden Türme in den Himmel wie zwei Arme und ahmte mit der Form der Glockentürme zwei gegeneinander gelegte, gefaltete Hände mit den zehn gestreckten Fingern nach, die Geste, die die Bildhauer voriger Zeiten den Heiligen und toten Kriegern auf ihren steinernen Sarkophagen verliehen haben“. Auguste Rodin stellte in der Plastik „La Cathedrale“ (1908) zwei Hände dar, die sich wie zu einem gotischen Spitzbogen oben zusammenschließen.

Die so oft als höherer Sinn der Kathedrale beschworene Frömmigkeit hatte schon in der Romantik ihre alte Bedeutung verloren. In der Zeit, als sich demokratische Staatsformen verbreiteten und die Sozialwissenschaften aufkamen, faszinierte an der Gotik besonders die Massenbewegung, in der sich alle Glieder der Gesellschaft zu einem einheitlichen Willen zusammenzuschließen schienen. Dieser "grenzenlose Gemeinschaftswille“ (Arthur Wachsberger, 1919) prägte das gesamte Bild von der Epoche der Pilgerreisen und Kreuzzüge. Die neuen Ergebnisse der Kunstgeschichte bestätigten ihn: Er fand seinen Ausdruck in dem einheitlichen „Kunstwollen“ ganzer Epochen, ganzer Völker (Alois Riegl, 1901). Er überwand die Ländergrenzen, wie die Ausbreitung der Gotik oder die Wanderungen von Architekten und Steinmetzen zeigten. Er überwand alle sozialen Schranken beim Bau der Kathedrale, wie der berühmte Bericht des Abtes Haimo von St-Pierre-sur-Dives bezeugt: „Wer sah jemals, wer hörte von vergangenen Generationen, dass Tyrannen, Fürsten, Mächtige, die in dieser Welt an Reichtümern und Ehren ganz aufgebläht sind, dass Frauen und Männer adliger Geburt, ihre stolzen und von Einbildung geschwollenen Häupter gebeugt und sich vor die Riemen der Karren gespannt haben und sie, beladen mit Wein, Getreide, Öl, Kalk, Steinen Holz und anderen zum Leben oder Bauen nützlichen Dingen zum Zufluchtsort Christi wie Zugvieh zogen? Das ist aber an ihnen wunderbar zu sehen, dass, obwohl ihrer tausend oder mehr, Frauen und Männer an die Karren gebunden sind [...], doch unter so großem Schweigen marschiert wird, dass man niemandes Stimme oder nur ein Flüstern hörte" (1145).

Der kulturkritische Tenor der Romantik lebte unter neuem Vorzeichen im frühen 20. Jahrhundert fort. Viele Architekten sahen die Gotik als Ausdruck einer Haltung an, die untergegangen war und neu belebt werden sollte. Walter Gropius gründete 1919 das Bauhaus, um die „Wiedergeburt jener Geisteseinheit, die sich zur Wundertat der gotischen Kathedrale aufschwang", zu erwirken. Deshalb ist auf dem Manifest des Bauhauses eine gotische Kathedrale dargestellt. Dazu schrieb Gropius: „Das ganze Volk baute, gestaltete, das war seine vornehmste Tätigkeit, das Handel treiben war sekundär. So war es in Deutschland in der besten Zeit der Gotik und so muss es bei uns wieder werden". Gropius wollte eine moderne Form der gotischen Bauhütte schaffen. Er rief sämtliche Künstler auf, mitzuringen um „das letzte Ziel der Kunst: die schöpferische Konzeption der Zukunftskathedrale, die wieder alles in einer Gestalt sein wird, Architektur und Plastik und Malerei“'. Alle Arten von Künstlern und Handwerkern sollten gemeinsam ausgebildet werden, so wie sie einst 
zusammengewirkt hatten, um das „,Gesamtkunstwerk“ der Kathedrale zu schaffen, die Plastiken und Bilder und viele andere Artefakte unter dem Dach der Architektur vereinte. Das Bauhaus projizierte in die gotische Bauhütte gesellschaftliche Ideale, die schon seit Langem verbreitet waren, sich aber nun teilweise dem Sozialismus annäherten.

Die gotische Bauhütte erschien als ein Ort besonderer Freiheit: Sie löste bürgerliche Gebote und Zunftzwänge. Architekten und Steinmetzen unterlagen nur den Regeln der Bauhütten und waren frei, sich dahin zu wenden, wo sie wollten. Sie sprengte scheinbar die Barrieren zwischen Wissenschaft und praktischer Tätigkeit, zwischen Akademikern, Künstlern und Handwerkern. Die Regeln ließen zu, dass einfache Steinmetzen zu leitenden Architekten aufsteigen konnten. Schon in der Aufklärung hatte die Bauhütte Intellektuelle angezogen. In England entstand der Bund der Freimaurer, der sich als Nachfolger der Bauhütte verstand. Ihm traten zahlreiche prominente Persönlichkeiten bei.

Gropius wollte den oft beklagten, Graben, der sich zwischen Kunst und Volk aufgetan hatte, überwinden. Der Architekt sollte die „Volksstimmung“ wiedergeben. Die Trennung von Kunst und Handwerk, die sich seit der Renaissance durchgesetzt hatte, sollte, wie überhaupt alle „klassentrennende Anmaßung“, rückgängig gemacht werden. Der Maler Franz Marc forderte schon 1915 die Abkehr von dem Geniekult, der mit der Renaissance eingesetzt hatte, und die Rückbesinnung auf die mittelalterlichen Verhältnisse: „Die namenlosen gotischen Meister - das sind die reinsten.[... ] Die Kunst ging an der vergiftenden Krankheit des Individualitätskultus zu Grunde, am Wichtignehmen des Persönlichen, an der Eitelkeit, davon muss man gänzlich loskommen“. Die Vorstellung vom anonymen Künstler des Mittelalters basiert darauf, dass nördlich der Alpen wenige Namen von Künstlern überliefert und die wenigen so schlecht dokumentiert sind, dass sie schwer mit Werken verbunden werden können. Dass der gemeinschaftliche Wille stärker war als der Drang nach Selbstverwirklichung einzelner Individuen, schien sich in der langen Bauzeit der Kathedralen und in der ganzen Stilentwicklung der Hochgotik zu bestätigen, die fast so einheitlich wirkt, als hätte ein einziger Architekt immer weiter hinzugelernt. Die Vorstellung übergeht allerdings Berichte vom hohen sozialen Status der Architekten oder die Grabmäler in französischen Kathedralen, die Architekten in vornehmem Habitus zeigen. Auch die Vorstellungen vom Architekten als anonymer Hand des Volkswillens haben romantische Wurzeln.

Immer wieder wurde die gotische Kathedrale wegen ihrer kühnen Konstruktion, ihrer filigranen Struktur, ihrer kristallinen Struktur mit geometrischer Grundlage, wegen ihrer Lichtfülle und ihrer Wirkung mit der modernen Glas-Eisen-Architektur verglichen. Derartige Vergleiche setzten sogleich ein, als die Glas-Eisen-Architektur prominent in Erscheinung trat, nämlich mit dem so genannten Kristallpalast der Londoner Weltausstellung von 1851 (Joseph Paxton). Umgekehrt führte der Philosoph Adolf Trendelenburg zwei Jahre später in einer Rede auf den Kölner Dom den Kristallpalast zum Vergleich an. Allerdings erging es der Glas-Eisen-Architektur anfangs ähnlich wie früher der Gotik: Ihr wurde kategorisch der Rang von Kunst abgesprochen. Sie schien lediglich auf praktische Zwecke Rücksicht zu nehmen und den flüchtigen Ansprüchen der Mode zu folgen. Dagegen galt für die Kunst nach wie vor die Devise, dass sie aus dem Alltag durch eine höhere Bestimmung von überzeitlichem Wert herausragen sollte. Trendelenberg und der Kunsthistoriker August Reichensperger stellten den Kristallpalast und den Kölner Dom in diesem Sinn einander gegenüber. Während der Kristallpalast mit seinem ,unabsehbaren Gewimmel von Formen und Farben“ und seiner „Durchsichtigkeit nach allen Seiten hin“ trotz seiner utilitären Sachlichkeit einen berauschenden Wirbel von äußerlichen Reizen erzeuge, führe der Dom durch seine „schweigsame, mit unbestimmtem Farbenschimmer durchleuchtete Atmosphäre“ zu innerer Einkehr und erwecke „die schlummernde Ahnung des Göttlichen“. Dabei wurden allerdings inzwischen bekannte Berichte übergangen, die überliefern, dass in den Domen manchmal 
mehr buntes Getriebe als Kontemplation herrschte. Bernhard von Clairvaux meinte, Bischöfe würden ihre Dome opulent gestalten, um ,die Andacht des fleischlich gesinnten Volkes mit materiellem Schmuck anzuregen, weil sie es mit Geistigem nicht vermögen".

Während die deutschen Avantgardisten zu Beginn des 20. Jahrhunderts nur eine bessere Zukunft in der Rückbesinnung auf die Gotik erhofften, hatte der Aufbruch für Le Corbusier bereits begonnen, allerdings nicht in Europa. In einer Schrift mit dem Titel „Als die Kathedralen weiß waren" (1935/36) setzte er die Wolkenkratzer von New York in Parallele zur gotischen Kathedrale, dem „Wolkenkratzer Gottes“. Die Kathedrale zeichne sich ebenfalls durch neue Techniken aus, durch die Kühnheit der Konstruktion und die Erfindung einer ganz neuartigen Formensprache, die strikt geometrischen Regeln folge. Sie sei hervorgegangen aus der freudigen Aufbruchstimmung, die die gesamte Bevölkerung einer neuen, befreiten Gesellschaft ergriffen habe. In einer ähnlichen Situation des jugendlichen Erwachens befänden sich jetzt die Vereinigten Staaten von Amerika. „Als die Kathedralen weiß waren" meint, als sie noch nicht den Staub romantischer Sehnsüchte und akademischer Theoreme angesetzt hatten. Die kulturkritischen Aspekte beim Rückblick auf die Gotik sind verflogen. Damit eröffnete sich die Möglichkeit, in der Erhebung zu Gott und im Rausch des Konsums die gleiche massenhafte „Begeisterung“ wahrzunehmen. So erkannte der französische Philosoph Roland Barthes schon 1957 im Auto „das genaue Äquivalent der großen gotischen Kathedralen, eine Schöpfung der Epoche, die mit Leidenschaft von unbekannten Künstlern erdacht wurde und [...] von einem ganzen Volk benutzt wird, das sich in ihr ein magisches Objekt zurüstet und aneignet“"

\section{Literaturhinweise}

Magdalena Bushart, Der Geist der Gotik und die expressionistische Kunst. München 1990. Paul FrankL, The Gothic. Literary Sources and Interpretations through Eight Centuries. Princeton 1960.

Michael Hesse, Von der Nachgotik zur Neugotik. Die Auseinandersetzung mit der Gotik in der französischen Sakralarchitektur des 16., 17. und 18. Jahrhunderts. Frankfurt/M./Bern/ New York 1984.

Hermann Hipp, Studien zur „Nachgotik“ des 16. und 17. Jahrhunderts in Deutschland, Böhmen, Österreich und der Schweiz. Diss. Tübingen 1979.

Dieter KIMPEL/Robert Suckale, Die gotische Architektur in Frankreich 1130-1270. München 1985.

Klaus NiEHR, Gotikbilder - Gotiktheorien. Studien zur Wahrnehmung und Erforschung mittelalterlicher Architektur in Deutschland zwischen ca. 1750 und 1850. Berlin 1999.

Monika Steinhauser, Geschichte im Dienste der Baukunst. Zur historischen ArchitekturDiskussion in Deutschland, in: „Geschichte allein ist zeitgemäß“. Historismus in Deutschland, hrsg. von Michael BrIx/Monika SteInHAuser. Lahn/Gießen 1978, S. 199-327.

Hermann STURM, Industriearchitektur als Kathedrale der Arbeit. Geschichte \& Gegenwart eines Mythos, Essen 2007.

Ludger J. Sutthoff, Gotik im Barock. Zur Frage der Kontinuität des Stiles außerhalb seiner Epoche. Möglichkeiten der Motivation bei der Stilwahl. Münster 1990.

Christoph WAGNER, Gotikvisionen am Bauhaus, in: Mittelalter und Mittelalterrezeption. Festschrift für Wolf Frobenius, hrsg. von Herbert ScHneIDE. Hildesheim 2005, S. 382-406. 